\title{
Nature des colonies se développant à la surface du milieu Violet Rouge Bile Lactose Agar (VRB) ensemencé avec du lait cru. Validité des dénombrements des colonies lactose-positives et des colonies lactose-négatives se développant sur ce milieu
}

\author{
J. RICHARD *, C. PITON ** et J.J. GRATADOUX * \\ * INRA, Laboratoire de Microbiologie laitière, 78350 Jouy-en-Josas, France \\ *** INRA, Station expérimentale laitière, 39800 Poligny, France
}

\section{Résumé}

Environ 140 échantillons de lait cru contenant entre $10^{4}$ et plus de $10^{7}$ ucf par ml ont été ensemencés à la surface du milieu Violet Rouge Bile Lactose Agar (VRB, Difco). Après $24 \mathrm{~h}$ d'incubation à $30^{\circ} \mathrm{C}$ puis autant à température ambiante, 8 colonies lactose-positives et 8 colonies lactose-négatives ont été prélevées sur chaque boîte de Petri et soumises à différents tests rapides d'identification.

Dans environ $95 \%$ des cas, la flore lacto-positive a pu être identifiée à des entérobactéries. Les autres souches de ce groupe appartenaient au genre Aeromonas. Les Pseudomonas du groupe fluorescent dominaient très nettement la flore lactosenégative dans la majorité des échantillons de lait, sauf dans ceux contenant moins de $10^{3}$ bactéries lactose-négatives où c'était le genre Acinetobacter qui l'emportait, et ceux qui en contenaient plus de $10^{6}$, où des entérobactéries devenaient de plus en plus fréquentes.

Il n'y avait pas de différence significative, pour le dénombrement des Pseudomonas psychrotrophes du lait cru, entre le milieu VRB et un milieu non sélectif, et la croissance de ces bactéries n'était pas affectée par le développement, sur le milieu VRB, de bactéries coliformes isolées du lait cru. Réciproquement, ces dernières se développaient normalement sur ce milieu en présence de ces Pseudomonas, quel qu'en soit le niveau. Par conséquent, l'ensemencement du lait cru à la surface du milieu VRB peut permettre la numération simultanée des bactéries coliformes (colonies lactosepositives) et des colonies lactose-négatives. Dans la limite où ces dernières sont comprises entre $10^{3}$ et $10^{6}$ par $\mathrm{ml}$, il s'agit essentiellement de Pseudomonas psychrotrophes.

Mots clés : Lait cru - Bactéries coliformes - Alcaligenes - Acinetobacter - Aeromonas Pseudomonas - Identification - Numération - Milieu de culture. 


\section{Summary}

Nature of the colonies growing on the surface of Violet Red Bile Agar inoculated with raw milk. Reliability and signification of the counting of lactose-negative and lactosepositive colonies present on this medium

About 140 samples of raw milk, ranging from $10^{4}$ to up to $10^{7} \mathrm{cfu} / \mathrm{ml}$ were submitted to a vigorous shacking for $30 \mathrm{~s}$ using a blender rotating at $20,000 \mathrm{rpm}$ to break the bacterial clumps. They were then surface plated on VRB without a second layer of medium. After $24 \mathrm{~h}$ of incubation at $30^{\circ} \mathrm{C}$ and one day at room temperature, 8 lactose-positive and 8 lactose-negative colonies were picked at random from each plate and characterized using the following tests: morphology and motility, Gram reaction ( $\mathrm{KOH}$ method), detection of a cytochrome-oxidase $c$, metabolism of glucose and growth at $2{ }^{\circ} \mathrm{C}$. In addition, the lactose-positive strains were tested for nitrate reduction.

More than $95 \%$ of the lactose-positive isolates were classified as enterobacteria, the remaining belonging to the genus Aeromonas.

The majority of the lactose-negative bacteria were identified as psychrotrophic Pseudomonas. However, in samples containing less than $10^{3}$ lactose-negative bacteria/ $\mathrm{ml}$ the genus Acinetobacter was predominant, followed by the genus Alcaligenes. On the other hand, lactose-negative enterobacteria predominated in some samples containing more than $10^{6}$ lactose-negative bacteria/ $\mathrm{ml}$.

Nutrient agar and VRB agar gave similar results in the enumeration of psychrotrophic Pseudomonas, and these bacteria were not affected by the growth of coliform bacteria on VRB and vice versa.

It is concluded that coliforms bacteria (lactose-positive colonies) and lactosenegative bacteria could be enumerated on VRB inoculated on surface with raw milk. When these lactose-negative bacteria are in the range of $10^{3}-10^{6} \mathrm{ufc} / \mathrm{ml}$, they probably belong to the psychrotroph group of Pseudomonas.

Key-words : Raw milk - Enterobacteria - Alcaligenes - Acinetobacter - Aeromonas Identification - Enumeration - Medium.

\section{Introduction}

Les méthodes de dénombrement des bactéries coliformes dans les denrées alimentaires ont fait l'objet de nombreuses études comparatives. Celles-ci ont abouti à des recommandations ou à des normes qui définissent le type de milieu sélectif utilisé, la manière de s'en servir et d'interpréter les résultats (Anon., 1974b, 1978 a et b, 1985). Pour les produits laitiers (Anon., 1974b), on a le choix entre un milieu liquide, le bouillon bilié au vert brillant et un milieu solide, la gélose lactosée biliée au cristal violet et au rouge neutre (VRBL, ou plus simplement VRB). La technique prévoit qu'après ensemencement et solidification de la gélose, on coule à sa surface, une couche supplémentaire de même milieu, sans doute pour inhiber les microorganismes aérobies stricts qui pourraient s'y développer. Cette manière de faire est difficilement compatible avec l'utilisation de la méthode "Spirale » d'ensemencement en surface de la gélose, dans laquelle l'inoculum (de l'ordre de $50 \mu \mathrm{l}$ ), est déposé de façon décroissante et rigoureusement répétitive, le long d'une spire 
d'Archimède (GiLCHRIST et al., 1973) : malgré les précautions que l'on peut prendre, l'étalement d'une seconde couche de milieu entraîne une partie de l'inoculum, ce qui fausse les résultats du dénombrement par la méthode «Spirale». Compte tenu de l'intérêt de celle-ci (facilité d'exécution, gain de temps et de matériel) il nous a paru intéressant de voir si cette deuxième couche était absolument nécessaire dans le cas du lait cru ; en d'autres termes, il fallait déterminer l'importance numérique et éventuellement la nature de la flore non coliforme se développant à la surface du milieu VRBL, et voir si le développement de cette flore influençait celui des bactéries coliformes. Une telle étude ne semble pas avoir été réalisée auparavant, ou en tout cas, avoir fait l'objet de publications récentes.

\section{Matériel et méthode}

\section{A. Nature des colonies lactose-négatives et lactose-positives}

\section{Dénombrements}

Environ 140 échantillons de lait cru de qualité bactériologique variable (de $10^{4}$ à plus de $10^{7}$ ucf par ml) ont été examinés. Il s'agissait de lait non réfrigéré livré au plus $2 \mathrm{~h}$ après la traite, de lait non réfrigéré âgé de 12 à $16 \mathrm{~h}$, ou de lait réfrigéré à la ferme (mélange de 2 à 4 traites).

Les échantillons ont été d'abord traités $30 \mathrm{~s}$ à l'aide d'une turbine ULTRA-TURRAX tournant 20000 tours/mn, de façon à disperser au maximum les amas microbiens (RICHARD, 1980b ; PITON et RichaRD, 1983), puis ensemencés à la surface du milieu VRB (Difco, 12) soit manuellement à l'aide d'une pipette Pasteur coudée (échantillons supposés contenir moins de $10^{3}$ bactéries coliformes $/ \mathrm{ml}$ ), soit à l'aide d'un ensemenceur en spirale (Spiral System Inc. ; modèle A). Après 16 à $18 \mathrm{~h}$ d'incubation des boîtes à $30^{\circ} \mathrm{C}$, on a compté les colonies lactose-positives, et après $24 \mathrm{~h}$ supplémentaires à température ambiante, les colonies lactose-négatives.

\section{Caractérisation des colonies lactose-négatives}

Sur chaque boîte de Petri, on a prélevé au hasard, suivant une méthode rapide (Richard, 1981a), 8 colonies lactose-négatives. On n'a pas jugé utile de les purifier avant leur caractérisation, car avec l'agitation par turbine, moins de $5 \%$ des agrégats de bactéries à Gram négatif contiennent plus de deux cellules (RICHARD, 1980b) ; la probabilité pour qu'une colonie bien isolée sur la boîte de Petri soit pure est donc très élevée.

On a effectué ensuite des suspensions de ces colonies dans les alvéoles d'une plaque de microtitration stérile contenant $0,2 \mathrm{ml}$ de bouillon stérile trypticase soja (TSB, Difco). Cette plaque a servi à ensemencer les milieux suivants, à l'aide d'un inoculateur multiple (Multipoint Inoculator, Denley, Grande-Bretagne) : 
- VRB, pour confirmation de croissance et du caractère lactose-négatif (milieu en boîte de Petri de $14 \mathrm{~cm}$ ),

- Nutrient Agar (NA, Difco), 3 boîtes de Petri de $14 \mathrm{~cm}$.

La plaque microtitre, la boîte de Petri contenant du VRB et 2 de celles contenant du NA étaient incubées 18 à $24 \mathrm{~h}$ à $30^{\circ} \mathrm{C}$, l'autre boîte de NA, 10 jours à $2{ }^{\circ} \mathrm{C}$; on effectuait ensuite les tests suivants :

a) Morphologie et mobilité des cellules ayant cultivé 16 à $18 \mathrm{~h}$ à $30^{\circ} \mathrm{C}$ sur TSB, Une variante rapide de la technique des gouttes pendantes (LowIS, 1969) a été utilisée : celle-ci consistait dans le dépôt d'une goutte de 4 cultures à la fois sur une lamelle de microscope de $24 \times 60 \mathrm{~mm}$; après avoir retourné la lamelle et l'avoir placée sur un support rigide, l'examen était fait en série, à l'aide d'un microscope à contraste de phase muni d'un objectif à immersion,

b) Coloration de Gram ou réaction à une solution de potasse : un fragment de colonie était mélangé avec une goutte de solution de $\mathrm{KOH}$ à $3 \%$. La formation d'un gel en 5 à $20 \mathrm{~s}$ correspond à une réaction de Gram négative (Buck, 1982),

c) Voie de métabolisme du glucose par la méthode à un seul tube (PARK et Holding, 1966). Le milieu de base (O/F, Difco) était bouilli pendant au moins 15 min pour éliminer l'oxygène dissous, puis il était additionné d'une solution de glucose stérilisée par filtration (concentration finale $10 \mathrm{~g} / \mathrm{l}$ ). Après mélange sans incorporation d'air, on répartissait le milieu en clochettes de Durham stériles (2 $\mathrm{ml}$ par tube). Celles-ci étaient ensemencées aussitôt en piqûres profondes par rang de 8 à la fois, à l'aide d'un "peigne » flexible sur lequel étaient montés 8 fils parallèles de ferro-nickel de 7 à $8 \mathrm{~cm}$ de longueur (Richard, 1981a). L'inoculum était prélevé sur l'une des boîtes de Petri contenant le milieu NA. Les tubes étaient examinés après 14 à 16 h d'incubation à $30^{\circ} \mathrm{C}$,

d) Mise en évidence d'une cytochrome-oxydase $c$ par la méthode rapide de GABY et HADLEY (1957). On préparait, extemporanément, une solution à $1 \%$ dans l'eau de tétraméthyl-p-phénylène diamine et une solution à $1 \%$ dans l'alcool à $90^{\circ} \mathrm{GL}$ de naphtol-1. On recouvrait les colonies présentes sur une boîte de NA par un mélange volume à volume des deux réactifs. Après 5 à $10 \mathrm{~s}$, on notait comme positives les colonies qui devenaient bleu pâle ou bleu foncé. Les colonies négatives étaient reprises pour confirmation sur l'autre boîte de NA et testées par la méthode de Kovacs (disques Institut Pasteur Production).

\section{Caractérisation des colonies lactose-positives}

De même façon simplifiée, miniaturisée et automatisée, on a soumis les colonies lactose-positives prélevées dans une cinquantaine d'échantillons (8 par échantillon) aux 4 tests suivants déjà décrits :

a) voie de métabolisme du glucose (test $\mathrm{O} / \mathrm{F}$ ),

b) morphologie et mobilité à l'état frais,

c) réaction de Gram (méthode par coloration ou par $\mathrm{KOH}$ ),

d) détection d'une cytochrome-oxydase $c$.

En outre, on a mis en évidence la réduction du nitrate après $48 \mathrm{~h}$ de culture sur bouillon nitrate (Nitrate Broth, Difco) réparti en clochette de 
Durham ( $2 \mathrm{ml} /$ tube). La présence de nitrite était révélée de façon classique par le N, N-diméthyle-1-naphtylamine en milieu acide (BLAzEvic et EdERER, 1975). Une réaction négative peut être due soit à l'absence de réduction du nitrate (souche nitrate-négative), soit à une réduction du nitrate au-delà des nitrites. Dans ce cas, les tubes ne devraient plus contenir de nitrate. La présence éventuelle de nitrate dans les tubes négatifs était donc recherchée par la réduction de ce dernier en nitrite, grâce à l'addition de poudre de zinc. En cas de réaction positive, on considérait la souche comme réellement négative ; le cas contraire correspond à une réduction du nitrate au-delà des nitrites, et la souche était donc réellement nitrate-positive.

\section{B. Effet de la croissance, à la surface du milieu VRB, de bactéries lactose-positives et de bactéries latose-négatives sur le nombre de colonies de chaque type}

Pour voir dans quelle mesure le développement des colonies lactosenégatives influençait celui des colonies lactose-positives, et réciproquement, on a réalisé des essais de croissance simultanée sur le milieu VRB de bactéries coliformes et de Pseudomonas, le genre lactose-négatif le plus fréquent lorsque le milieu est ensemencé avec du lait cru (voir résultats).

\section{Origine des souches}

Vingt-deux souches représentatives des différents types de Pseudomonas psychrotrophes ont été utilisées. Ces souches provenaient du matériel de traite, de la peau des mamelles ou du lait cru après conservation prolongée à basse température (RICHARD, 1980a).

Dix souches de bactéries coliformes (5 de Escherichia coli et 5 de Hafnia alvei, variétés lactose-positives), isolées de camemberts fabriqués à partir de lait cru, ont également été utilisées. Ces deux espèces ont été choisies car ce sont les plus fréquentes dans le lait cru (RICHARD et BraquenaYe, 1985). Ces souches ont été cultivées sur le milieu Plate Count Agar (PCA, Difco) juste avant leur emploi.

2. Comparaison des nombres de colonies de Pseudomonas obtenues sur le milieu $V R B$ et sur le milieu de référence non inhibiteur

Pour voir dans quelle mesure le milieu VRB est inhibiteur vis-à-vis des Pseudomonas, une colonie de chaque souche de cette espèce était prélevée sur le milieu PCA et diluée dans du lait entier stérile de façon à obtenir des suspensions bactériennes contenant $10^{4}$ à $10^{6}$ cellules $/ \mathrm{ml}$. Ces suspensions étaient ensuite soumises à un dénombrement en surface sur milieu Violet Red Bile Agar (VRB, Difco) et sur milieu Nutrient Agar (NA, Difco). L'inoculum était étalé à la surface de la gélose à l'aide de l'ensemenceur en spirale, après avoir été traité $30 \mathrm{~s}$ à l'Ultra-Turrax. Après $24 \mathrm{~h}$ d'incubation à $30^{\circ} \mathrm{C}$ plus un jour à température ambiante, on procédait au comptage des colonies dans l'une des 4 zones concentriques (correspondant à un volume connu d'inoculum), dans laquelle ces colonies étaient bien isolées (nombres généralement inférieurs à 200). 
3. Croissance simultanée des Pseudomonas et des bactéries coliformes sur le milieu $V R B$.

Une colonie de chaque souche de Pseudomonas et de chaque souche de bactéries coliformes a été diluée dans du lait entier stérile de façon à obtenir des suspensions bactériennes à un « haut niveau » de population $\left(10^{5}\right.$ à $10^{6}$ cellules/ml) ; une dilution à $1 / 100$ permettait d'avoir des suspensions à « faible niveau » de population. On réalisait ensuite, pour chaque couple de souches (une souche de Pseudomonas et une souche d'entérobactérie), un mélange, volume à volume, de ces suspensions, de façon à obtenir 4 suspensions de cultures mixtes (l'une étant, par exemple, une suspension contenant une souche de Pseudomonas à « haut niveau » et une E. coli à «faible niveau»). On effectuait enfin, par la méthode Spirale, le dénombrement sur le milieu VRB des bactéries lactose-positives et lactose-négatives présentes dans ces suspensions et dans les cultures pures d'origine diluées à $1 / 2$ (témoins).

\section{Résultats}

A. Caractérisation des colonies se développant à la surface du milieu VRB ensemencé avec du lait cru

Toutes les colonies prélevées sur le milieu VRB ont donné un gel avec la potasse à $3 \%$. On peut donc les considérer comme étant Gram-négatives. On a d'ailleurs confirmé, par sondage sur environ $10 \%$ des souches isolées, qu'elles ne prenaient effectivement pas la coloration de Gram.

Les colonies lactose-négatives provenant de près de 120 échantillons ont été classées de la façon suivante (Anon., 1974a) :

\begin{tabular}{l|c|c|c|c|c}
\hline & Test O/F & Morphologie & Mobilité & $\begin{array}{c}\text { Cytochrome- } \\
\text { oxydase }\end{array}$ & $\begin{array}{c}\text { Nitrate- } \\
\text { réductase }\end{array}$ \\
\hline Pseudomonas & $\mathrm{O}(\mathrm{a})$ & $\mathrm{B}(\mathrm{b})$ & + & + & $\mathrm{NC}(\mathrm{c})$ \\
Acinetobacter & $\mathrm{A}$ & $\mathrm{CB}$ & - & - & $\mathrm{NC}$ \\
Alcaligenes & $\mathrm{A}$ & $\mathrm{B}$ & + & + & $\mathrm{NC}$ \\
Entérobactéries & $\mathrm{F}$ & $\mathrm{B}$ & $\mathrm{NC}$ & + & + \\
Vibrionacées & $\mathrm{F}$ & $\mathrm{B}$ & + & + & $\mathrm{NC}$ \\
\hline
\end{tabular}

(a) : O : Oxydatif ; A : Alcalinisant ; F : fermentatif.

(b) : B : Bâtonnet; $\mathrm{CB}$ : CoccoBacilles.

(c) : NC: non pris en compte (résultats variables).

Toutes les colonies classées dans le genre Pseudomonas cultivaient à $2{ }^{\circ} \mathrm{C}$, alors que celles classées dans le genre Acinetobacter en étaient incapables. Cela confirme des résultats antérieurs (RICHARD, 1981b).

La figure 1 montre que les Pseudomonas dominaient la flore lactosenégative qui poussait sur le milieu VRB, dans la majorité des échantillons de 


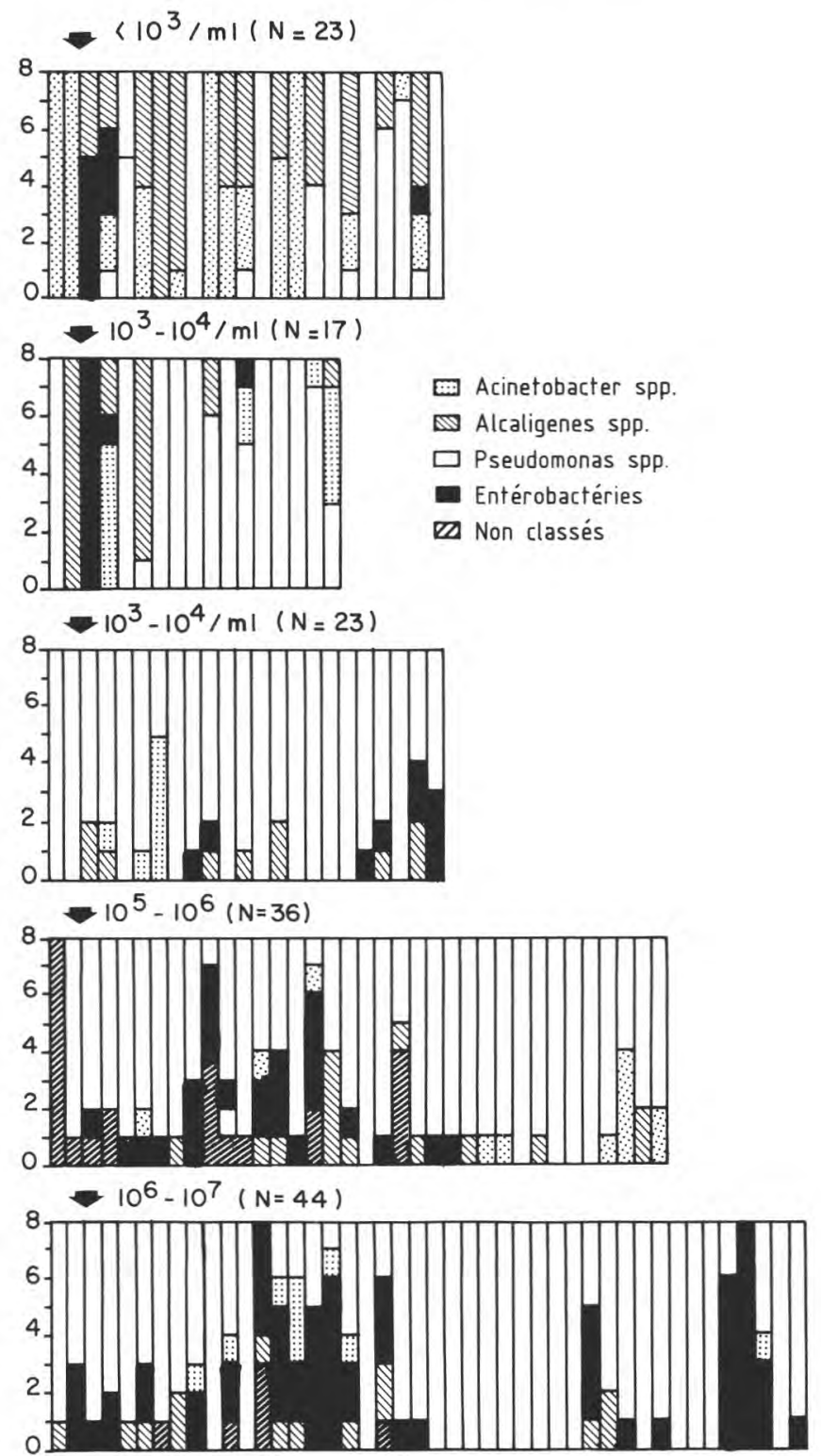

Fig. 1

Fréquence des divers types de bactéries lactose-négatives sur le milieu VRB, en fonction de cette population dans le lait cru. (En ordonnée : nombre de colonies caractérisées; en abscisse: échantillons différents).

Incidence of different types of lactose-negative bacteria growing onto the surface of Violet Red Bile Agar inoculated with raw milk after $1 \mathrm{~d}$ of incubation at $30^{\circ} \mathrm{C}$ and $1 \mathrm{~d}$ at room temperature. (Vertical axis : no of characterized colonies per sample; horizontal axis : samples of milk from different origins). 
lait, sauf dans ceux contenant moins de $10^{3}$ bactéries lactose-négatives par $\mathrm{ml}$, où c'était le genre Acinetobacter qui l'emportait, suivi du genre Alcaligenes.

Des entérobactéries lactose-négatives n'ont été détectées que dans un faible pourcentage d'échantillons. Elles paraissaient plus souvent présentes en flore dominante dans le lait très pollué (plus de $10^{5}$ bactéries lactose-négatives par $\mathrm{ml}$ ). De très rares souches ont été classées dans les vibrionacées. Quelques colonies n'ont pas pu être classées; d'autres n'ont pas donné de culture après repiquage.

Pour 45 échantillons sur 50 (soit $90 \%$ des cas), les 8 colonies lactosepositives prélevées appartenaient aux entérobactéries. Dans le cas de 2 échantillons, les 8 colonies isolées appartenaient aux vibrionacées (bâtonnets fermentatifs et oxydase-positifs). Dans les autres cas, 1 à 2 colonies seulement sur 8 , appartenaient à cette famille. Trois souches de celle-ci, provenant de 3 échantillons différents, ont été identifiées à l'espèce Aeromonas hydrophila, à l'aide de galeries API $20 \mathrm{E}$ incubées à $30^{\circ} \mathrm{C}$.

B. Effet de la croissance, à la surface du milieu VRB, de bactéries lactose-positives et de bactéries lactose-négatives sur le nombre de colonies de chaque type

1. Comparaison des nombres de colonies de Pseudomonas sur le milieu VRB et sur un milieu non sélectif

La figure 2 présente les nombres de colonies de Pseudomonas par $\mathrm{ml}$ de suspensions bactériennes sur le milieu non sélectif et sur le milieu VRB. On

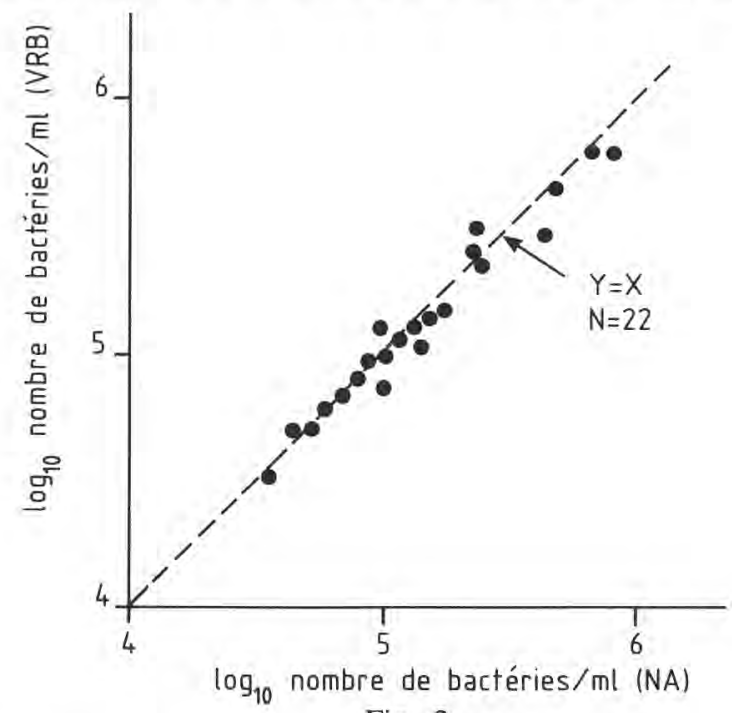

Fig. 2

Comparaison des milieux Nutrient Agar (NA) et Violet Rouge Bile Lactose Agar (VRB) pour le dénombrement à $30^{\circ} \mathrm{C}$ de Pseudomonas psychrotrophes en cultures pures. (Dénombrements effectués sur plusieurs souches de cette espèce, à des niveaux différents).

Comparison of Nutrient Agar (NA) and Violet Red Bile Agar (VRB) for the enumeration at $30^{\circ} \mathrm{C}$ of psychrotrophic Pseudomonas in pure cultures. (Enumeration using 22 different strains at different levels of contamination). 
n'observe aucune différence entre ces deux milieux, les écarts à la droite d'équation $\mathrm{Y}=\mathrm{X}$ pouvant être attribués à l'erreur d'analyse. Le test $\mathrm{t}$ de Student réalisé sur ces données n'était d'ailleurs pas significatif (SNEDECOR et Cochran, 1957). Par contre, le diamètre des colonies du VRB était habituellement inférieur à celui observé sur NA.

\section{Croissance simultanée des Pseudomonas et des bactéries coliformes sur le milieu $V R B$}

Le tableau 1 permet de comparer les nombres moyens de colonies de Pseudomonas et de bactéries coliformes obtenus sur VRB ensemencé avec des suspensions pures et des suspensions mixtes de ces deux groupes de bactéries.

Le comptage des colonies a été réalisé sur toute la boîte pour les « faibles niveaux » ou seulement dans la couronne où les nombres de colonies étaient compris entre 30 et 200 pour les « hauts niveaux». Cependant, dans le cas particulier des suspensions contenant les Pseudomonas à un "faible niveau » et les bactéries coliformes à un «haut niveau» ( $3^{\text {e }}$ ligne du tableau 1$)$, le comptage des Pseudomonas (colonies lactose-négatives) n'a pu être effectué que dans les couronnes les plus externes, car ces colonies n'étaient pas répérables dans la masse des colonies lactose-positives ayant poussé plus au centre de la boîte ; c'est pourquoi les nombres moyens de colonies de Pseudomonas sont faibles.

On observe que les nombres moyens de colonies de Pseudomonas ou de bactéries coliformes obtenus avec les suspensions pures et avec les suspensions mixtes de même concentration, sont très voisins, quel que soit le niveau de l'un ou l'autre groupe microbien. Nous avons voulu vérifier par le test $t$ de Student (SNEdeCor et Cochran, 1957), si les écarts obtenus étaient, en moyenne, significativement différents ou non de zéro. Cependant, étant donné que les nombres de colonies sur une boîte de Petri suivent approximativement une loi de Poisson, il en résulte que l'intervalle de confiance d'une donnée varie en fonction du nombre de colonies. Pour tenter de rendre gaussienne la distribution des écarts, afin de pouvoir appliquer le test $t$ sans trop de biais, nous avons pondéré les écarts entre les nombres obtenus en culture pure (Np) et en culture mixte $(\mathrm{Nm})$ par celui obtenu en culture pure :

$$
\text { écarts pondérés }=\frac{\mathrm{Np}-\mathrm{Nm}}{\mathrm{Np}}
$$

Ces écarts, en moyenne, n'étaient pas significativement différents de zéro $(\mathrm{P}<0,05)$.

\section{Discussion et conclusions}

Plusieurs études ont montré que la méthode spirale d'ensemencement à la surface des milieux de culture donnait des résultats qui différaient généralement peu de ceux de la méthode par dilutions et incorporation de l'inoculum dans ces mêmes milieux (CAMPBell et Gilchrist,1973 ; Gilchrist et al., 1973 ; Peeler et al., 1977 ; Jarvis et al., 1977 ; Kramer et al., 1979 ; Catteau et al., 1981). En particulier, Gilchrist et al. (1973) ont montré, avec des cultures pures de divers microorganismes anaérobies facultatifs, dont Escherichia coli, 


\section{TABLEAU 1}

Comparaison des nombres moyens de colonies de Pseudomonas et de bactéries coliformes obtenues à la surface du milieu VRB ensemencé en Spirale à partir de suspensions pures et de suspensions mixtes de ces bactéries

Comparison of the average colony numbers of Pseudomonas and coliform bacteria growing on the surface of VRB inoculated with pure and mixed cultures of these two groups of bacteria

\begin{tabular}{|c|c|c|c|c|c|c|}
\hline \multirow{2}{*}{\multicolumn{2}{|c|}{$\begin{array}{l}\text { Niveau de population } \\
\text { des suspensions }\end{array}$}} & \multirow{4}{*}{$\mathrm{N}$} & \multicolumn{4}{|c|}{ Nombre moyen de colonies } \\
\hline & & & \multirow{2}{*}{\multicolumn{2}{|c|}{$\begin{array}{c}\text { Pseudomonas } \\
\text { Suspensions }\end{array}$}} & \multirow{2}{*}{\multicolumn{2}{|c|}{$\begin{array}{c}\text { Bactéries coliformes } \\
\text { Suspensions }\end{array}$}} \\
\hline \multirow{2}{*}{ Pseudomonas } & \multirow{2}{*}{$\begin{array}{l}\text { Bactéries } \\
\text { coliformes }\end{array}$} & & & & & \\
\hline & & & pures & mixtes & pures & mixtes \\
\hline Faible & Haut & 29 & 8 & 8 & NC & $\mathrm{NC}$ \\
\hline Faible & Faible & 18 & 82 & 86 & 75 & 83 \\
\hline
\end{tabular}

$\mathrm{N}$ : Nombre de répétitions avec des souches différentes.

NC : Comptage non réalisé car les colonies étaient largement dominantes par rapport à l'autre groupe microbien. 
que la méthode spirale donnait un nombre de colonies supérieur d'environ $20 \%$ à celui de la méthode conventionnelle d'ensemencement dans la masse de la gélose. Cette différence, sans conséquence pratique, peut être expliquée, soit par l'effet nocif, sur des cellules souvent endommagées, de la gélose chaude utilisée dans la méthode conventionnelle, soit par le fait que les colonies de bactéries coliformes se développent mieux en surface que dans la gélose. C'est en effet ce qu'ont observé VAN Soestbergen et LeE (1969) et Weber et Hamann (1981). Cela explique sans doute pourquoi les bactéries coliformes donnent parfois des colonies d'un diamètre inférieur à $0,5 \mathrm{~mm}$ lorsqu'elles cultivent dans la masse de la gélose (HARTMAN, 1960 ; Jones et al., 1966). On peut donc admettre que le dénombrement des bactéries coliformes à la surface de la gélose donne des résultats équivalents, sinon peu supérieurs, à ceux de la méthode conventionnelle.

La présente étude a montré par ailleurs que les résultats n'étaient pas affectés par la croissance de Pseudomonas psychrotrophes, même lorsque ces bactéries sont largement dominantes. Cela sans doute parce que ces dernières n'utilisent pas les mêmes substrats que les bactéries coliformes, et qu'elles ne modifient pas sensiblement le $\mathrm{pH}$ du milieu.

Inversement, les Pseudomonas, qui dominent habituellement la flore lactose-négative du lait cru, ne sont pas entravés dans leur croissance sur le milieu VRB par celle de bactéries coliformes. Cela aurait pu se produire, du fait de l'acidification de ce milieu par les colonies lactose-positives. On pourrait donc utiliser le milieu VRB pour estimer le nombre de Pseudomonas présents dans le lait cru. Toutefois, cette estimation ne serait valable que pour les échantillons contenant entre $10^{3}$ et $10^{6}$ bactéries lactose-négatives par $\mathrm{ml}$. En deçà de cette limite, le genre Acinetobacter semble plus souvent dominer que le genre Pseudomonas. Au-delà on trouve, dans le lait non réfrigéré conservé plus de $12 \mathrm{~h}$, d'autant plus souvent des entérobactéries lactosenégatives en flore dominante que le niveau de la flore augmente. Il s'agit alors principalement de l'espèce $H$. alvei et secondairement, de l'espèce Serratia liquefaciens (RICHARD, 1984).

Accessoirement, la présente étude permet de remarquer que les entérobactéries lactose-négatives sont largement sous-dominantes dans le lait cru. II n'est donc pas étonnant que Weber et Hamann (1981) n'aient pas observé de différence significative entre le milieu VRBL, contenant du lactose, avec lequel on ne compte que les bactéries lactose-positives, et le milieu VRBG, contenant du glucose, avec lequel on dénombre toutes les entérobactéries.

Reçu le 30 avril 1987.

Accepté pour publication le 19 novembre 1987.

\section{Références bibliographiques}

Anonyme, 1974(a). Bergey's Manual of Determinative Bacteriology. R.E. Buchanan \& N.E. Gibbons (eds), The Williams \& Wilkins Company, Baltimore, USA.

Anonyme, 1974(b). Lait et produits laitiers. Dénombrement des bactéries coliformes. Méthode de routine $B(V R B)$. Fédération Internationale de Laiterie (FIL) Doc 73, Bruxelles.

Anonyme, 1978(a). Microorganisms in Food. 1. Their significance and methods of enumeration. ICMFS, University of Toronto. 
Anonyme, 1978(b). Microbiologie Directives générales pour le dénombrement des coliformes. Méthode par comptage des colonies obtenues à $30^{\circ} \mathrm{C}$. Organisation Internationale de Normalisation, ISO 4832.

Anonyme, 1985. Standard Methods for the Examination of Dairy Products. American Public Health Association, 15th ed., Washington, D.C.

Blazevic D.J., Ederer G.M., 1975. Principles of biochemical tests in diagnostic microbiology. A Wiley Biomedical Publication, John Wiley \& Sons, New York.

BUCK J.D., 1982. Nonstaining (KOH) method for determination of Gram reaction of marine bacteria. Appl. Environ. Microbiol., 44, 992-993.

CAmpbell J.E., Gilchrist J.E., 1973. Spiral plating for counting bacteria in milk and other foods. Dev. Ind. Microbiol., 14, 95-102.

Catteau M., Auby J.C., Catsaras M., 1981. System spiral et analyse bactériologique des plats cuisinés. Rec. Méd. Vét., 157, 5 p.

GaBy W.L., Hadley C., 1957. Practical laboratory test for the identification of Pseudomonas aeruginosa. J. Bacteriol., 74, 356-358.

Gilchrist J.E., Campbell J.E., Donnelly C.B., Peeler J.T., Delaney J.M., 1973. Spiral plate method for bacterial determination. Appl. Microbiol., 25, 244-252.

HARRigan W.F., McCANCE M.E., 1976. Laboratory methods in food and dairy microbiology. Academic Press, London.

Hartman P.A., 1960. Further studies on the selectivity of Violet Red Bile Agar. J. Milk Food Technol., 23, 45-48.

JARVIS B., LACH V.H., WooD J.M., 1977. Evaluation of the spiral plate maker for the enumeration of microorganisms in foods. J. Appl. Bacteriol., 42, 149-157.

Jones G.A., Gibson D.L., Cheng K.J., 1966. Characterization of bacteria which produce colonies atypical of the coliform group on Violet Red Bile Agar. J. Milk Food Technol., 29, 316-318.

Kramer J.M., Kendall M., Gilbert R.J., 1979. Evaluation of the spiral plate and laser colony counting technique for the enumeration of bacteria in foods. Eur. J. Appl. Microbiol., 6, 289299.

Lowis M.J., 1969. A comparison of methods used to determine motility of strains of gramnegative bacteria isolated from aqueous pharmaceutical preparations. Lab. Pract., 18, 929-932.

PARK R.W.A., Holding A.J., 1966. Identification of some common Gram-negative bacteria. Lab. Pract., 15, 1124-1127.

Peeler J.T., Gilchrist J.E., Donnelly C.B., Campbell J.E., 1977. A collaborative study of the spiral plate method for examining milk samples. J. Food Prot., 40, 462-464.

Prton C., Richard J., 1983. Influence de l'agitation des échantillons de lait cru sur les résultats de dénombrement de trois groupes microbiens. Lait, 63, 405-415.

Richard J., 1980(a). Taxonomy and ecology of raw milk psychrotrophic Pseudomonas. In : Psychrotrophic Microorganisms in Spoilage and Pathogenicity. T.A. Roberts, G. Hobbs, J.H.B. Christian, N. Skovgaard (eds), Academic Press, London, 117-125.

Richard J., 1980(b). Influence de l'agitation du lait cru sur les résultats de dénombrement de sa flore totale à l'aide d'une anse calibrée. Lait, 60, 211-225.

Richard J., 1981(a). Rapid picking up and purifying of aerobic microorganisms. Lab. Pract., 30, $342-344$.

RICHARD J., 1981(b). Influence de diverses méthodes de nettoyage des machines à traire sur la qualité de conservation du lait cru à basse température. Lait, 61, 354-369.

Richard J., 1984. Evolution de la flore microbienne à la surface des camemberts fabriqués avec du lait cru. Lait, 64, 496-520.

Richard J., Braquehaye C., 1985. Les bactéries coliformes du lait cru. Sci. Aliments, 5, $\mathrm{n}^{\circ}$ huis série, 21-24.

Snedecor G.W., Cochran W.G., 1957. Statistical methods. The Iowa State University Press, Ames, Iowa, USA.

Van Soestbergen A.A., Lee C.H., 1969. Pour plates or streak plates? Appl. Microbiol., 18, 1092-1093.

Weber H., Hamann R., 1981. Bestimmung von coliformen Bakterien oder Enterobacteriaceen zur Beurteilung des Hygienestatus von Rohmilch. Dairy Sci. Abstr., 43, 710. 